\title{
The Influence of Litter Size on Brain Damage Caused by Hypoxic-Ischemic Injury in the Neonatal Rat
}

\author{
ELIZABETH OAKDEN, MALCOLM CHISWICK, NANCY ROTHWELL, AND SARAH LODDICK \\ School of Biological Sciences, University of Manchester, Manchester, U.K. [E.O., N.R., S.L.]; St Mary's \\ Hospital, Manchester, U.K. [M.C.]
}

\begin{abstract}
ABST
Hypoxic ischemia is a common cause of brain injury in the
human neonate. This can be mimicked in the neonatal rat, but
produces variable injury. The present study investigated the
influence of litter size on the severity and variability of damage
caused by hypoxic-ischemic injury in neonatal rats. Groups of
7-d-old pups from birth-sized litters (13-15 pups), or from litters
culled to 10 on postnatal d 2 , and 8 - and 9-d-old pups from
birth-sized litters, were exposed to common carotid artery occlu-
sion and then, $3 \mathrm{~h}$ later, hypoxia ( $2 \mathrm{~h} 15$ min, $8 \%$ oxygen).
Damage was assessed histologically $72 \mathrm{~h}$ after injury, and graded
(I-IV) according to severity. In nonculled litters, similar numbers
of animals had each grade of injury. Most pups (70\%) from
culled litters had grade III or IV damage, and severity was
\end{abstract}
ABSTRACT

significantly greater than in nonculled litters $(p<0.001)$. Pups from culled litters were heavier $(17.6 \pm 0.4 \mathrm{~g})$ than pups from nonculled litters $(14.7 \pm 0.3 \mathrm{~g}, p<0.0001)$. To determine whether this indicated that culled litters were more similar to older pups in their response to hypoxic-ischemic injury, we examined injury in 8- and 9-d-old pups of similar body weight to 7-d-old pups from culled litters. The severity and distribution of damage in the older pups was different from damage in the 7-d-old pups from culled litters. These data suggest that in 7-d-old rats, litter size influences damage caused by hypoxicischemic injury, and that the relationship between body weight, brain development, and susceptibility to hypoxic-ischemic injury is complex. (Pediatr Res 52: 692-696, 2002)
Hypoxic-ischemic injury in neonates occurs as a result of intrauterine hypoxia and birth asphyxia and is a significant cause of death and disability $(1,2)$. To study the mechanisms underlying hypoxic-ischemic injury in the laboratory setting, the injury can be mimicked in the neonatal rat (3).

A commonly used animal model to study perinatal hypoxicischemic brain damage is the 7-d-old rat subjected to unilateral common carotid ligation followed by exposure to $8 \%$ oxygen $(4,5)$.

The severity of damage caused by hypoxic-ischemic injury in the 7-d-old rat is variable, often requiring 25-30 animals per group, and a similar number of controls, to test treatments and interventions (6-9). Variation is reportedly limited by using pups of similar body weight (10-12), and several studies have culled litters, possibly to reduce variation in body weight (10, $13,14)$, but no universal protocol has been followed. A previous study observed that pups assigned to litters of six on

Received December 28, 2000; accepted June 26, 2002.

Correspondence: Nancy Rothwell, School of Biological Sciences, Room 1.124, Stopford Building, University of Manchester, Oxford Road, Manchester, M13 9PT, U.K.; e-mail: nancy.rothwell@man.ac.uk

Supported by Central Manchester Healthcare Trust, Medical Research Council, and The Royal Society.

DOI: 10.1203/01.PDR.0000032070.60058.BB postnatal d 1 (P1), were more susceptible to injury on $\mathrm{P} 7$ than those from litters of 14 (6). However, in this study, damage was assessed by measurement of brain size.

Thus, the aim of the present study was to investigate in more detail the effect of culling litters on the damage caused by hypoxic-ischemic injury in the 7-d-old rat, and to determine the relationship between body weight and injury severity in culled and nonculled litters. Pups from culled litters were found to be heavier than those from birth-sized litters. By comparing damage in culled pups with older pups of a similar weight from birth-sized litters, we tested the hypothesis that susceptibility to hypoxic-ischemic injury in culled litters was influenced by maturity and body size.

\section{METHODS}

Animals. Time-mated, pregnant female rats (Sprague Dawley, Charles River, Margate, Kent, U.K.) were housed individually in a temperature-controlled $\left(19-22^{\circ} \mathrm{C}\right)$, artificially lit (0800-2000 h) environment, and provided with food (CRM, Labsure, Poole, Dorset, U.K.) and water ad libitum. Pups were delivered spontaneously, and the day of birth was defined as P0. Pups remained with the dam until surgery. All surgical procedures were performed in accordance with the U.K. Animals (Scientific Procedures) Act, 1986. 
Hypoxic-ischemic injury. Four groups of animals were exposed to hypoxic ischemia: 7-d-old pups from birth-sized litters ( $n=33$, mean litter size 14, range 13-15); 7-d-old pups from litters that had been culled to 10 pups on P2 $(n=41)$; and 8- $(n=24)$ and 9-d-old $(n=32)$ pups, both from birth-sized litters. On the day of the experiment, pups were anesthetized with halothane $(3-4 \%$ for induction and $0.75-1.5 \%$ for maintenance) (Fluothane, Zeneca Pharmaceuticals plc, Macclesfield, U.K.) in a mixture of nitrous oxide and oxygen (70\%:30\%). An incision was made medially on the left side of the neck, and the left common carotid artery was exposed and isolated from the adjacent vagus nerve and jugular vein under a dissecting microscope. The artery was then ligated (silk suture, 5/0: Davis and Geck, Basingstoke, U.K.) sectioned between the ligatures and the wound sutured (Mersilk sutures, 6/0 Johnson and Johnson, Skipton, U.K.). The total duration of anesthesia was $<20 \mathrm{~min}$. Animals were returned to the dam and allowed to recover for at least $3 \mathrm{~h}$ in their normal environment. In sham-operated animals, the common carotid artery was exposed, the wound closed, and the pups were returned to the dam until the injured littermates were killed.

The injured pups (6-12) were placed into a humidified, temperature-controlled chamber. This consisted of a 2-L glass Buchner flask partially immersed in a water bath maintained at $36.5 \pm 0.5^{\circ} \mathrm{C}$. The flask was supplied with humidified air, pumped through water in a second Buchner flask, also immersed in the water bath. A humidified mixture of precalibrated gas $8.1 \%$ oxygen; $91.9 \%$ balance nitrogen (BOC Gases, Guilford, U.K.) was used to perfuse the chamber for $2.25 \mathrm{~h}$ in place of air. Samples of the gaseous mixture within the flask were collected periodically and analyzed (Servomex Group, Crowborough, East Sussex, U.K.). Oxygen levels were maintained at $8.1 \pm 0.1$. Immediately after the hypoxic-ischemic insult, the animals were returned to their respective dams. During procedures and recovery, animals were kept at an ambient temperature of $22-23^{\circ} \mathrm{C}$.

Assessment of damage. Seventy-two hours after hypoxicischemic injury, animals were anesthetized in halothane, killed by cervical dislocation, and their brains were removed. The brains were frozen in isopentane $\left(-25\right.$ to $\left.-30^{\circ} \mathrm{C}\right)$ and stored briefly at $-20^{\circ} \mathrm{C}$. Tissue was embedded in Tissue-Tek (Dental Supplies, Orpington, U.K.) and $20-\mu \mathrm{m}$ coronal sections (taken every $450 \mu \mathrm{m}$ ) were cut on a freezing microtome (Leica, Wetzlar, Germany) before mounting on 3-aminopropyltriethoxysilane (APES)-coated slides.

Air-dried sections (nine per animal) were stained with hematoxylin and eosin, dehydrated in descending concentrations of alcohol, cleared in xylene, and mounted in mounting medium, DePEX (Raymond Lamb, Eastbourne, U.K.), under a coverslip. Once dry, the slides were studied using a slide viewer and light microscope. Damage was defined as regions where eosinophilic staining was pale and nuclear densities were not uniform - either shrunken and condensed, absent, or pale and enlarged. Areas of damaged and nondamaged tissue were recorded onto stereotaxic brain maps (taken from ref. 15) at $900-\mu \mathrm{m}$ intervals in the coronal plane, and the severity of the damage for each individual animal was graded (Fig. 1). The assessor was blind to the treatment. A second blind observer

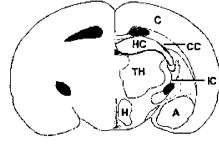

1

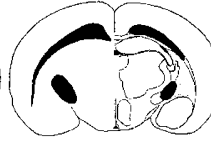

II

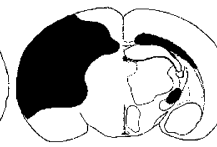

III

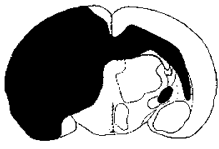

IV
Figure 1. Grading scale. Diagrams are of coronal sections of 10-d-old rat brain (injured at 7-d-old) at the level of the internal capsule (IC) and hippocampus $(H C)$. The borders of the region of damaged tissue for each grade (I-IV), according to severity, are represented by the shaded areas. Contralateral damage was observed in animals injured at 7-d-old and some of the animals injured at 8-d (39\%) and 9-d (13\%). TH, thalamus; $A$, amygdala.

assessed 12 randomly selected brains and recorded levels of damage with $95 \%$ consistency with the first observer.

Grading scale. In 7-d-old and some 8- (39\%) and 9-d-old $(13 \%)$ animals, damage appeared bilaterally, normally with larger areas of tissue damage on the ipsilateral side. Assessment was based on the nature of the ipsilateral damage only.

Grade I was defined as pale eosinophilic staining (also seen in controls) together with disruption of regular tissue architecture, (including irregular distribution of nuclear hematoxylin stain) in the body of the corpus callosum and the posterior limb of the internal capsule, compared with surrounding tissue.

Grade II was defined as further disruption of tissue as shown by pale eosinophilic staining, and irregular hematoxylin staining in the arm of the corpus callosum, with enlargement of damage in the internal capsule.

Grade III was defined as disruption of the corpus callosum and hippocampal damage indicated by loss of deeply stained (basophilic) cells in the CA1/CA3 regions; some regions of neuronal loss were seen in the ipsilateral cortex with $>5 \%$ of the ipsilateral cortex damaged and $<35 \%$ of the ipsilateral subcortical tissue damaged.

Grade IV was defined as infarction in most $(>75 \%)$ of the ipsilateral hemisphere (including both cortical and subcortical areas) and definite hippocampal ablation ipsilaterally.

Statistical analysis. Differences in damage between the groups were examined using $\chi^{2}$ analysis. Body weights were analyzed using $t$ test and Welch's correction. The relationship between body weight and brain damage was analyzed using a Pearson correlation. Data are expressed as mean and SE.

\section{RESULTS}

Severity of hypoxic-ischemic injury. No damage was seen in either naïve $(n=5)$ or sham-operated animals $(n=6)$. All pups from nonculled litters subjected to hypoxic-ischemic injury exhibited brain damage, which varied from mild (grade I, $n=10$ ) to severe infarction (grade IV, $n=9$ ) (Figs. 1 and 2). Typically, damage in the corpus callosum and internal capsule was characterized by regions of pale staining and disruption of structure (Figs. 2 and 3). In animals with more severe grades of injury, damage extended laterally in the corpus callosum, into the hippocampus, and from the internal capsule into the surrounding tissue; as severity increased this became continuous with the damaged lateral portion of the corpus callosum. In more severely damaged brains, disruption of tissue architecture extended further into the overlying cortex and ventrally from the corpus callosum and the internal capsule 

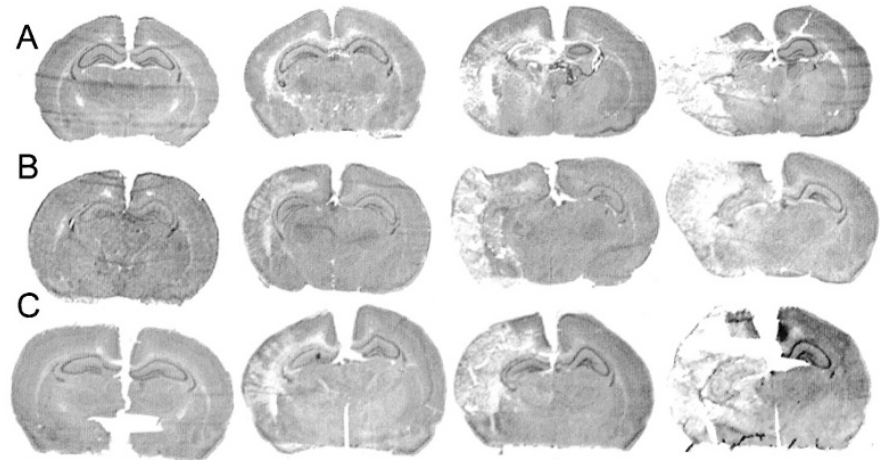

- 1

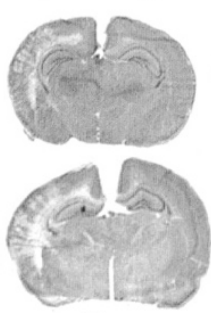

II

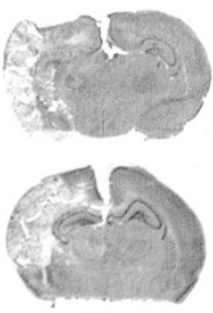

III

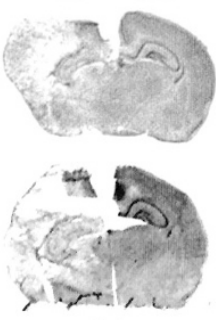

IV

Figure 2. Grades of brain damage $72 \mathrm{~h}$ post hypoxic-ischemic injury in nonculled litters. Typical hematoxylin and eosin stained coronal $(20 \mu \mathrm{m})$ sections. All sections were taken at the level where the internal capsule and the hippocampus were both visible. On P7 $(A)$, P8 $(B)$, or P9 $(C)$, animals from birth-sized litters were subjected to unilateral common carotid artery ligation and section. They were allowed to recover from surgery and then exposed to $2 \mathrm{~h} 15 \mathrm{~min}$ in an $8 \%$ oxygen environment kept at $36-37^{\circ} \mathrm{C}$. Scale bar $=1.8$ $\mathrm{mm}$.

into thalamic tissue. The most severely affected animals exhibited complete cortical infarction of the hemisphere, with tissue damage in subcortical structures throughout the entire rostrocaudal axis (Fig. 2).

Influence of litter size on brain damage. As described above, animals from nonculled litters (containing 13-15 pups) subjected to hypoxic ischemia presented with variable damage, which was distributed almost evenly across all four grades of injury (Fig. 4A). In contrast, the majority (37/41) of the animals from culled litters (10 pups/litter) presented with severe damage (grade III or IV), only four animals presented with grade II damage (Fig. 4A). Statistical analysis confirmed the distribution of damage in culled litters was significantly different from nonculled P7 litters $\left(\chi^{2}, p<0.001\right)$. Of the 75 animals used in the study, one died during hypoxia and four died during surgery.

Relationship between body weight and brain damage. In the same population, the relationship between body weight and brain damage was examined. Seven-day-old pups from culled litters were significantly $(p<0.0001)$ heavier (range 12.5-23.5 g) than pups from nonculled litters $(12.3-17.7 \mathrm{~g}, p<0.0001)$. There was no relationship between body weight and the grade of damage in pups from nonculled litters. In culled litters, there was a correlation between body weight and injury severity, with heavier pups having more severe damage ( $p<0.05$, Fig. $4 B)$.

Hypoxic-ischemic injury in 8- and 9-d-old litters. To determine whether the severe damage observed in the heavier, culled pups reflected enhanced maturity, we examined the effect of injury in 8-d-old pups from nonculled litters, in which body weight was similar to those from 7-d-old culled litters, and in 9-d-old animals from nonculled litters, in which body weights were above the 75 th percentile of 7-d-old pups. There was no correlation between body weight and injury in either the 8-d-old pups or the 9-d-old pups, and in both groups damage was variable and distributed almost equally across all four grades of injury. The pattern of white matter injury in the
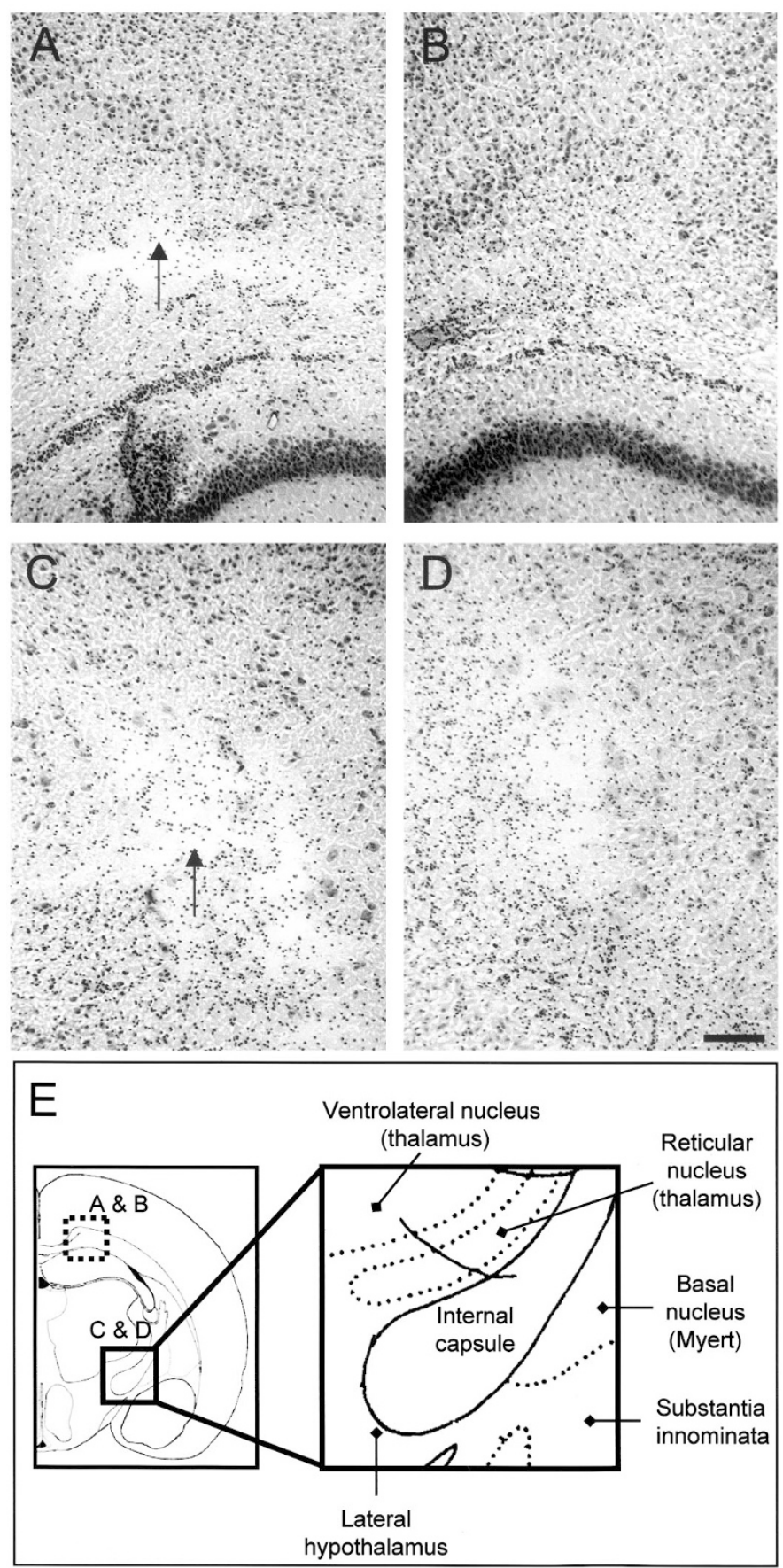

Figure 3. Grade I damage in regions of white matter. Coronal $20 \mu \mathrm{m}$ sections stained with hematoxylin and eosin taken from injured $(A, C)$ and naïve $(B, D)$ rat brains at the level of the posterior limb of the internal capsule. $E$ indicates the regions represented by $A$ and $B$ (indicated by dotted-line square) and $C$ and $D$ (indicated by solid-line square). On P7, animals were subjected to unilateral common carotid artery ligation and section, allowed to recover from surgery, and then exposed to $2 \mathrm{~h} 15 \mathrm{~min}$ in an $8 \%$ oxygen environment, kept at $36-37^{\circ} \mathrm{C}$. Myelinogenic foci in the corpus callosum, $A$ and $B$; internal capsule, $C$ and $D$. Orientation: top is dorsal and bottom is ventral. Pale, damaged regions are indicated by arrows. The damage in this injured brain $(A$ and $C)$ is classified as grade I. Scale bar $=200 \mu \mathrm{m}$.

older (8-d and 9-d) animals was also different from the 7-d animals. All 7-d-old pups subjected to hypoxic-ischemic injury had bilateral damage in the corpus callosum and the internal capsule (regions containing fiber tracts of myelinated axons, 

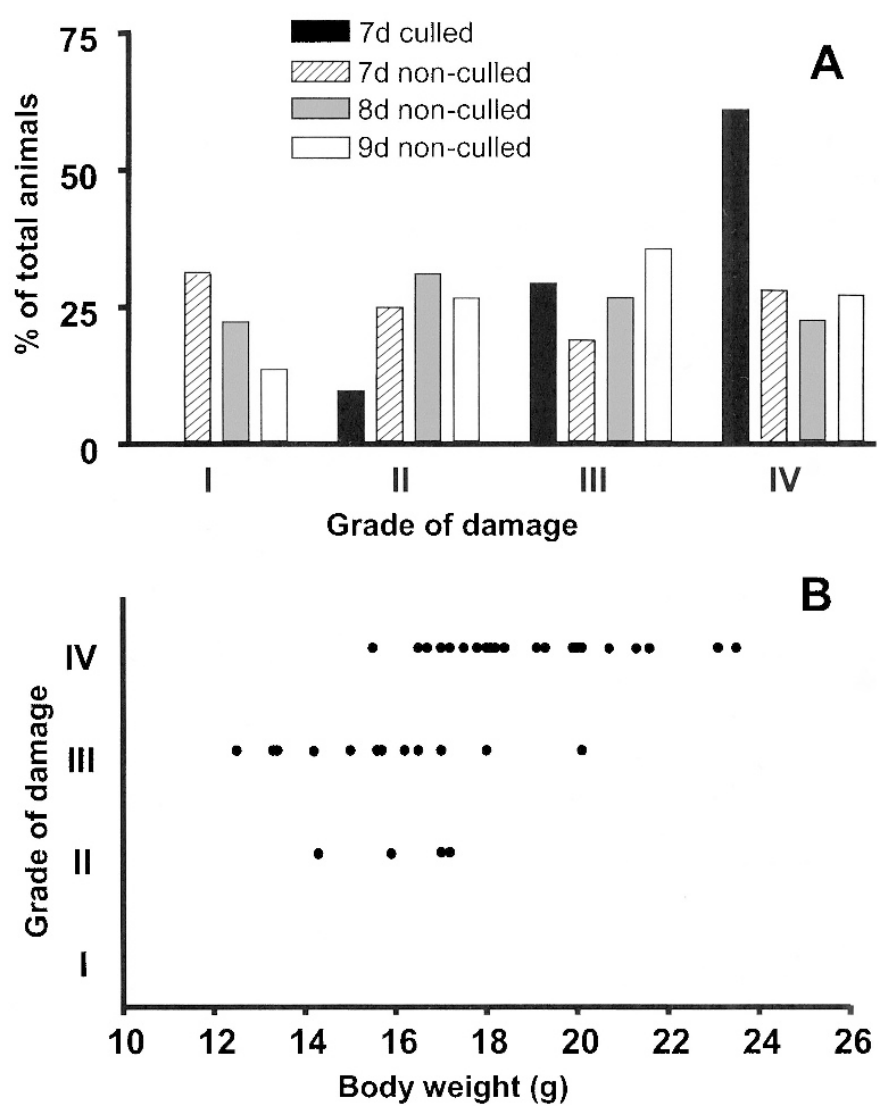

Figure 4. Severity of damage caused by hypoxic-ischemic injury. (A) Severity of damage in pups from culled and nonculled litters injured on P7 and in pups from nonculled litters injured on P8 and P9. The distribution of damage in P7 culled litters was significantly different from nonculled P7 litters $(p<$ 0.001). Animals were subjected to unilateral common carotid artery ligation and section, allowed to recover from surgery, and then exposed to $2 \mathrm{~h} 15 \mathrm{~min}$ in an $8 \%$ oxygen environment, kept at $36-37^{\circ} \mathrm{C}$. $(B)$ Grade of damage and body weight. Level of damage is plotted against body weight (g) pups from culled litters injured on P7. There was a correlation between body weight and injury severity, with heavier pups having more severe damage $(p<0.05)$.

i.e. white matter). In 8-d-old pups, the bilateral damage was less frequent $(39 \%)$ and in 9 -d-old pups was absent in the majority $(87 \%)$ of animals.

Of the 56 animals used in this study, 10 died during hypoxia.

Hypoxic-ischemic interval. In the same experiment, the time between occlusion of the carotid artery (i.e. onset of ischemia) and entry into the hypoxic chamber was recorded and varied between animals $(3-5.7 \mathrm{~h})$. Subsequent analysis revealed that the length of this interval had no relationship to the degree of damage (data not shown).

\section{DISCUSSION}

Standard hematoxylin and eosin histologic staining was used to differentiate between viable and nonviable tissue. This has advantages over other commonly used methods such as measurement of brain mass, edema, neuronal excitability in damaged regions, or behavioral outcomes (16-19), which do not permit analysis of cellular or regional damage, or reveal subtle changes in tissue or cellular integrity. To illustrate the variation in the extent of damage caused by hypoxic-ischemic injury, damage was assessed by categorizing severity into four bands. This has advantages over measurement of lesion volume inasmuch as hypoxic ischemia causes diffuse injury in selective regions. Furthermore, direct measurement is inaccurate where hemispheric swelling or shrinkage is associated with the lesion, so normally an indirect approach is used to calculate lesion volume (20). These methods assume the contralateral hemisphere is undamaged, with no swelling or shrinkage. We attempted to use such a method in the present study, and for animals (7-d-old) exhibiting extensive infarction (grade IV) calculated a lesion volume of $203 \pm 22 \mathrm{~mm}^{3}$. In the present study, however, we believe that this approach is misleading. As reported previously (12), there is damage and edema in the contralateral hemisphere, and although some animals had considerable swelling of the ipsilateral hemisphere (up to $140 \%$ of the contralateral volume), others had considerable shrinkage (reduction in volume of up to $40 \%$ ). Thus, the calculations of lesion volume are inaccurate. Therefore, in the present study, we used the method of grading injury severity as used previously $(21,22)$.

The variation in damage observed between pups from nonculled litters was consistent with data presented by Rice et al. (3), who noted that $56 \%$ of animals had a discrete area of infarction, as did $46 \%$ of all the 7 -d-old animals in the present study. The nature and cause of this variation is unknown. We have demonstrated that damage is maximal $72 \mathrm{~h}$ after injury (data not shown), so the variation observed within this experimental group was not because damage is still progressing, but could relate to other experimental and/or biologic variants.

In 7-d-old litters culled to 10 pups, damage was statistically different from nonculled pups, with most $(90 \%)$ animals exhibiting severe injury with cortical infarction, and hippocampal and thalamic damage. The fact that in nonculled litters animals exhibited all grades of damage, whereas those from culled litters exhibited mainly grade III and IV damage, indicates that variation was reduced in the latter. Pups of this age are capable of regulating body temperature independently of heat derived from the dam $(23,24)$. However, it is possible that pups from culled litters have a higher body temperature than those from birth-sized litters through spending more time in close proximity to the mother. Because hypothermia can protect the brain from hypoxic-ischemic injury (25-27), such an increase in body temperature could influence the extent of brain injury to cause more severe damage. We did not investigate this possibility in the present study as this would require repeated measurement of body temperature, which is associated with a stress response that would confound results. Pups from culled litters had a greater body weight $(17.6 \pm 0.4 \mathrm{~g})$ than those from the nonculled litters $(14.7 \pm 0.3 \mathrm{~g}, p<0.0001)$. There was a correlation between body weight and injury severity in the culled litters, but not the nonculled litters; thus, heavier animals appear to be more susceptible to injury. Pups from culled litters may be heavier due to greater milk availability, and it has been reported that reducing maternal food intake significantly impairs brain development (for review, see ref. 28). Thus, increased susceptibility to hypoxic-ischemic injury may reflect enhanced maturity (e.g. brain development) in the culled litters.

Several protective mechanisms may operate in younger animals, including higher efficiency of the oxygen transfer (29), and 
the ability to use lactate as a substrate for glycolysis (30) and to divert energy resources away from nonessential processes of growth $(31,32)$. These mechanisms are gradually lost as the animal matures (33). In younger animals, there is a lower density of ion channels, which limits damaging changes in membrane potential that can occur because of hypoxic-ischemic injury (34, $35)$. Furthermore, the number and distribution of excitatory amino acid receptors changes during development (36).

The hypothesis that the increased susceptibility of culled litters to hypoxic-ischemic brain damage was due to enhanced maturity was not supported by our comparison of damage in 7-d-old pups from culled litters with damage from older pups from birth-sized litters. The older animals (8-d-old) from nonculled litters had comparable body weights $(16.7 \pm 0.2 \mathrm{~g})$ to pups from 7-d-old culled litters, but the damage was variable; $20-30 \%$ had each grade of damage (Fig. 4A). These data suggest that the 7-d-old culled litters are not equivalent to 8-d-old nonculled litters, and that body weight is not the sole predictor of damage severity, though it is possible that age and body weight each has an independent effect on susceptibility to hypoxic ischemia. Furthermore, damage in pups from 9-d-old nonculled litters whose body weights $(19.2 \pm 0.4 \mathrm{~g})$ were comparable to the 75th quartile of the culled group was also variable (13-35\% having each grade, Fig. $4 A$ ).

There was a reduced incidence of contralateral white matter damage in the older (8-d and 9-d) animals compared with the 7-d-old animals (as described in the "Results" section). These findings are consistent with previous reports (37) and suggest that, in common with the human, the increased susceptibility of regions of white matter to injury becomes less pronounced with age. It is possible that detailed examination of all aspects of pup development would reveal differences between culled and nonculled litters. However, the different pattern and severity of damage observed in 8- and 9-d-old pups, compared with 7-d-old culled pups, suggests that the increased susceptibility to damage in the culled litters is not explained by a general enhancement of maturity, and is unlikely to be due to uniformly enhanced brain maturity.

Thus, reducing litter size by culling results in heavier pups that are more susceptible to hypoxic-ischemic injury. The mechanisms underlying the effect of litter size on susceptibility to hypoxic-ischemic injury are presently unknown. Body weight alone is not a reliable indicator of susceptibility to hypoxic-ischemic injury in birth-sized litters, and although culling litters may influence the development of pups, the present data suggest the effect on brain development is complex because damage severity and the pattern of white matter damage is different from the older pups.

Acknowledgment. The authors thank Dr. Rosemary Gibson for her advice in preparing this manuscript.

\section{REFERENCES}

1. Hull J, Dodd KL 1992 Falling incidence of hypoxic-ischaemic encephalopathy in term infants. Br J Obstet Gynaecol 99:386-391

2. Gonzalez DD, Moya M 1997 Perinatal differences in asphyxic full-term newborns in relation to the presence of hypoxic-ischemic encephalopathy. Rev Neurol 25:1187-1194

3. Rice JE, Vannucci RC, Brierley JB 1981 The influence of immaturity on hypoxicischemic brain damage in the rat. Ann Neurol 9:131-141
4. Silverstein F, Buchanan K, Johnston MV 1984 Pathogenesis of hypoxic-ischemic brain injury in a perinatal rodent model. Neurosci Lett 49:271-277

5. Dobbing J, Sands J 1979 Comparative aspects of the brain growth spurt. Early Hum Dev 3:79-83

6. Trescher WH, Lehman RA, Vannucci RC 1990 The influence of growth retardation on perinatal hypoxic-ischemic brain damage. Early Hum Dev 21:165-173

7. Towfighi J, Mauger D, Vannucci RC, Vannucci SJ 1997 Influence of age on the cerebral lesions in an immature rat model of cerebral hypoxia-ischemia: a light microscopic study. Dev Brain Res 100:149-160

8. Eun BL, Liu XH, Barks JD 2000 Pentoxifylline attenuates hypoxic-ischemic brain injury in immature rats. Pediatr Res 47:73-78

9. Hagberg H, Bona E, Gilland E, Puka-Sundvall M 1997 Hypoxia-ischaemia model in the 7-day-old rat: possibilities and shortcomings. Acta Paediatr Suppl 422:85-88

10. Cheng Y, Deshmukh M, D'Costa A, Demaro JA, Gidday JM, Shah A, Sun Y, Jacquin MF, Johnson EM, Holtzman DM 1998 Caspase inhibitor affords neuroprotection with delayed administration in a rat model of neonatal hypoxic-ischemic brain injury. J Clin Invest 101:1992-1999

11. Spandou E, Karkavelas G, Soubasi V, Avgovstides-Savvopoulou P, Loizidis T, Guiba-Tziampiri O 1999 Effect of ketamine on hypoxic-ischemic brain damage in newborn rats. Brain Res 819:1-7

12. Nedelcu J, Klein MA, Aguzzi A, Boesiger P, Martin E 1999 Biphasic edema after hypoxic-ischemic brain injury in neonatal rats reflects early neuronal and late glial damage. Pediatr Res 46:297-304

13. Lee WH, Wang GM, Seaman LB, Vannucci SJ 1996 Coordinate IGF-I and IGFBP5 gene expression in perinatal rat brain after hypoxia-ischemia. J Cereb Blood Flow Metab 16:227-236

14. Han BH, Holtzman DM 2000 BDNF protects the neonatal brain from hypoxicischemic injury in vivo via the ERK pathway. J Neurosci 20:5775-5781

15. Sherwood N, Timiras P 1970 A Stereotaxic Atlas of the Developing Rat Brain. University of California Press, Berkeley, CA, pp 14-69

16. Bona E, Johansson BB, Hagberg H 1997 Sensorimotor function and neuropathology five to six weeks after hypoxia-ischemia in seven-day-old rats. Pediatr Res 42:678-683

17. Balduini W, De A, V, Mazzoni E, Cimino M 2000 Long-lasting behavioral alterations following a hypoxic/ischemic brain injury in neonatal rats. Brain Res 859:318-325

18. Young RS, Kolonich J, Woods CL, Yagel SK 1986 Behavioral performance of rats following neonatal hypoxia-ischemia. Stroke 17:1313-1316

19. Jansen EM, Low WC 1996 Long-term effects of neonatal ischemic-hypoxic brain injury on sensorimotor and locomotor tasks in rats. Behav Brain Res 78:189-194

20. Swanson RA, Morton MT, Tsao-Wu G, Savalos RA, Davidson C, Sharp FR 1990 A semiautomated method for measuring brain infarct volume. J Cereb Blood Flow Metab 10:290-293

21. Hagberg H, Gilland E, Diemer NH, Andine P 1994 Hypoxia-ischemia in the neonatal rat brain: histopathology after post-treatment with NMDA and non-NMDA receptor antagonists. Biol Neonate 66:205-213

22. Schwartz PH, Massarweh WF, Vinters HV, Wasterlain CG 1992 A rat model of severe neonatal hypoxic-ischemic brain injury. Stroke 23:539-546

23. Schmidt I, Barone A, Carlisle HJ 1986 Diurnal cycle of core temperature in huddling, week-old rat pups. Physiol Behav 37:105-109

24. Alberts JR 1978 Huddling by rat pups: group behavioral mechanisms of temperature regulation and energy conservation. J Comp Physiol Psychol 92:231-245

25. Tomimatsu T, Fukuda H, Endo M, Watanabe N, Mu J, Kohzuki M, Fujii E, Kanzaki T, Murata Y 2001 Effects of hypothermia on neonatal hypoxic-ischemic brain injury in the rat: phosphorylation of Akt, activation of caspase-3-like protease. Neurosci Lett 312:21-24

26. Caputa M, Rogalska J, Nowakowska A 2001 Effect of temperature on postanoxic, potentially neurotoxic changes of plasma $\mathrm{pH}$ and free iron level in newborn rats. Brain Res Bull 55:281-286

27. Saeed D, Goetzman BW, Gospe Jr SM 1993 Brain injury and protective effects of hypothermia using triphenyltetrazolium chloride in neonatal rat. Pediatr Neurol 9:263-267

28. Thomas YM, Bedi KS, Davies CA, Dobbing J 1979 A stereological analysis of the neuronal and synaptic content of the frontal and cerebellar cortex of weaning rats undernourished from birth. Early Hum Dev 3:109-126

29. Metcalfe J, Bartels H, Moll W 1967 Gas exchange in the pregnant uterus. Physiol Rev 47:782-838

30. Fischer D, Heymann M, Rudolph A 1982 Fetal myocardial oxygen and carbohydrate consumption during acutely induced hypoxemia. Am J Cardiol 242:H657-H661

31. Adolph E 1969 Regulation during survival without oxygen in infant mammals. Respir Physiol 7:356-368

32. Weiser W 1984 A distinction must be made between ontogeny and the phylogeny of metabolism to understand the mass exponent of energy metabolism. Respir Physiol 55:1-9

33. Singer D 1999 Neonatal tolerance to hypoxia: a comparative-physiological approach Comp Biochem Physiol A Mol Integr Physiol 123:221-234

34. Xia Y, Haddad GG 1994 Postnatal development of voltage-sensitive $\mathrm{Na}+$ channels in rat brain. J Comp Neurol 345:279-287

35. Jiang C, Xia Y, Haddad GG 1992 Role of ATP-sensitive K+ channels during anoxia: major differences between rat (newborn and adult) and turtle neurons. J Physiol (Lond) 448:599-612

36. McDonald JW, Johnston MV 1993 Excitatory amino acid neurotoxicity in the developing brain. NIDA Res Monogr 133:185-205:185-205

37. Li YB, Kaur C, Ling EA 1998 Neuronal degeneration and microglial reaction in the fetal and postnatal rat brain after transient maternal hypoxia. Neurosci Res 32:137148 